\title{
Novel Role for the NMDA Receptor Redox Modulatory Site in the Pathophysiology of Seizures
}

\author{
Russell M. Sanchez, ${ }^{1}$ Carl Wang, ${ }^{1}$ Ginger Gardner, ${ }^{2}$ Lianna Orlando, ${ }^{3}$ David L. Tauck, ${ }^{4}$ Paul A. Rosenberg, ${ }^{1,3}$ \\ Elias Aizenman, ${ }^{5}$ and Frances E. Jensen ${ }^{1,3}$ \\ 1Department of Neurology, Children's Hospital and Harvard Medical School, Boston, Massachusetts, 2Department of \\ Obstetrics and Gynecology, Johns Hopkins Medical School, Baltimore, Maryland, ${ }^{3}$ Program in Neuroscience, Harvard \\ Medical School, Boston, Massachusetts, ${ }^{4}$ Department of Biology, Santa Clara University, Santa Clara, California, and \\ ${ }^{5}$ Department of Neurobiology, University of Pittsburgh School of Medicine, Pittsburgh, Pennsylvania
}

Redox-active compounds modulate NMDA receptors (NMDARs) such that reduction of NMDAR redox sites increases, and oxidation decreases, NMDAR-mediated activity. Because NMDARs contribute to the pathophysiology of seizures, redox-active compounds also may modulate seizure activity. We report that the oxidant 5,5'-dithio-bis(2-nitrobenzoic acid) (DTNB) and the redox cofactor pyrroloquinoline quinone (PQQ) suppressed low $\mathrm{Mg}^{2+}$ induced hippocampal epileptiform activity in vitro. Additionally, in slices exposed to 4-7 $\mu \mathrm{M}$ bicuculline, DTNB and PQQ reversed the potentiation of evoked epileptiform responses by the reductants dithiothreitol and Tris(2-carboxyethyl)phosphine (TCEP). NMDA-evoked whole-cell currents in CA1 neurons in slices were increased by TCEP and subsequently decreased by DTNB or $P Q Q$ at the same concentrations that modulated epileptiform activity. However, DTNB and PQQ had little effect on baseline NMDA-evoked currents in control medium, and PQQ did not alter NMDAR-dependent long-term potentiation. In contrast, in slices

The NMDA subtype of the glutamate receptor (NMDAR) critically contributes to the pathophysiology of seizures and epilepsy (Dingledine et al., 1990; Bradford, 1995; Chapman, 1998). Pharmacological inhibition of NMDARs is widely viewed as a potential therapeutic antiepileptic strategy, but the usefulness of this approach has yet to be established because of the adverse effects of NMDAR antagonists at therapeutic doses (Kornhuber and Weller, 1997; Chapman, 1998; Dannhardt and Kohl, 1998). A potential target of therapeutic NMDAR modulation is the redox modulatory site, because NMDAR function is potentiated by disulfide reductants and inhibited by sulfhydryl oxidants in vitro (Aizenman et al., 1989; Tang and Aizenman, 1993). Redox reagents are thought to induce conformational changes in the

\footnotetext{
Received Nov. 9, 1999; revised Dec. 29, 1999; accepted Jan. 4, 2000.

This research was supported by the Public Health Service National Institutes of Health and the National Institute on Aging Grants T32 AG00222 (National Research Service Award to R.M.S.), R01 NS31718 (F.E.J.), R01 NS29365 (E.A.), and R01 NS26830 (P.A.R.) and by the National Institutes of Health and the National Institute of Child Health and Human Development Mental Retardation Research Center Grant P30 HD18655. Additional support was provided by an Epilepsy Foundation of America research training fellowship with support from the Milken Family Medical Foundation (R.M.S.) and an Epilepsy Foundation of America research grant (F.E.J.). We thank Dr. Mercedes Paz for helpful comments and discussion in the preparation of this manuscript.

R.M.S. and C.W. contributed equally to this work.

Correspondence should be addressed to Dr. Frances E. Jensen, Department of Neurology, Enders 347, Children's Hospital, 300 Longwood Avenue, Boston, MA 02115. E-mail: jensen@a1.tch.harvard.edu.

Copyright (C) 2000 Society for Neuroscience $\quad 0270-6474 / 00 / 202409-09 \$ 15.00 / 0$
}

returned to control medium after low $\mathrm{Mg}^{2+}$-induced ictal activity, DTNB significantly inhibited NMDAR-mediated currents, indicating endogenous reduction of NMDAR redox sites under this epileptogenic condition. These data suggested that PQQ and DTNB suppressed spontaneous ictal activity by reversing pathological NMDAR redox potentiation without inhibiting physiological NMDAR function. In vivo, $\mathrm{PQQ}$ decreased the duration of chemoconvulsant-induced seizures in rat pups with no effect on baseline behavior. Our results reveal endogenous potentiation of NMDAR function via mass reduction of redox sites as a novel mechanism that may enhance epileptogenesis and facilitate the transition to status epilepticus. The results further suggest that redox-active compounds may have therapeutic use by reversing NMDAR-mediated pathophysiology without blocking physiological NMDAR function.

Key words: NMDA receptor; redox; epilepsy; hippocampus; patch clamp; $P Q Q$

NMDAR protein by acting at paired cysteine residues to promote or inhibit the formation of disulfide bridges with consequent changes in channel properties (Aizenman et al., 1989). This is supported by mutation studies that identified cysteine residues in cloned NMDAR subunits that are necessary for redox sensitivity (Kohr et al., 1994; Sullivan et al., 1994). Although the precise effects on channel behavior in recombinant NMDARs vary depending on which subunits are expressed (Brimecombe et al., 1997), chemical reduction of native and recombinant NMDARs generally causes increased channel-opening frequency relative to when the redox sites are oxidized (Tang and Aizenman, 1993; Brimecombe et al., 1997). Thus, reduction increases and oxidation decreases, but does not block, NMDAR-mediated activity. This incomplete inhibition of NMDAR function by oxidation of the redox site presents a possible mechanism for therapeutic NMDAR downmodulation without the adverse consequences of complete blockade.

The NMDAR redox site also may play a critical role in the expression of seizure activity. The accumulation of reducing equivalents in brain and serum is a prominent metabolic consequence of seizure activity, as demonstrated by increases in the NADH/NAD ${ }^{+}$ratio (Tenny et al., 1980; Dora, 1983; Fujikawa et al., 1988), ascorbic acid (Layton et al., 1998), and reduced cytochrome oxidase (Hoshi and Tamura, 1993). Additionally, prolonged status epilepticus can lead to brain hypoxia, and hypoxia is associated with an extracellular increase in reducing compounds 
such as reduced glutathione, cysteine, and related metabolites $(\mathrm{Li}$ et al., 1999). The reducing agent dithiothreitol (DTT) was shown to induce spontaneous epileptiform activity in guinea pig hippocampal slices (Tolliver and Pellmar, 1987). Because DTT increases NMDAR activity via reduction of the redox site (Aizenman et al., 1989), it is possible that such NMDAR potentiation contributed to the expression of DTT-induced epileptiform activity. Given these observations, we hypothesized that seizures may induce endogenous reduction of NMDAR redox sites, and the consequent potentiation of NMDAR function could sustain or prolong seizure activity.

In this study, we examined the ability of redox-active compounds to modulate epileptiform activity and NMDAR function in vitro under various epileptogenic conditions. Our aim was to determine the extent to which the NMDAR redox state contributes to the patterning of epileptiform activity and to determine whether NMDAR redox state is endogenously altered under epileptogenic conditions. Our results demonstrate that agents that oxidize the NMDAR redox site suppress experimentally induced epileptiform activity in vitro and inhibit behavioral seizures in vivo. More important, our data indicate that some epileptogenic conditions result in NMDAR potentiation by endogenous reducing factors. These findings suggest that the dynamic regulation of NMDAR function by the redox environment may be a critical mechanism in the expression of epileptiform activity and may therefore represent a target of therapeutic intervention.

\section{MATERIALS AND METHODS}

Animals. Long-Evans rats (Charles River Laboratories, Wilmington, MA) were housed in the animal care facility on a $12 \mathrm{hr}$ light/dark cycle. All procedures were approved and in accordance with guidelines set by the institutional Animal Care and Use Committee.

Extracellular recordings. Hippocampal slices were prepared according to our previously published protocol (Jensen et al., 1998) from rat pups aged postnatal day 10 (P10)-P18 or from adult rats $(250-300 \mathrm{gm})$. Younger rats were used for $0 \mathrm{Mg}^{2+}$ experiments, and both young and adult rats were used for low-bicuculline methiodide (BMI) experiments. No age-dependent differences were seen in the low-BMI results. After death by decapitation, the brain was rapidly dissected, sliced at $400 \mu \mathrm{m}$ thickness on a Stoelting tissue chopper, and transferred to an interface chamber continuously perfused with artificial CSF (ACSF) at $33.5^{\circ} \mathrm{C}$ at $38 \mathrm{ml} / \mathrm{hr}$ (in mM): $124 \mathrm{NaCl}, 5 \mathrm{KCl}, 1.25 \mathrm{NaH}_{2} \mathrm{PO}_{4}, 2 \mathrm{CaCl}_{2}, 1.5 \mathrm{MgSO}_{4}$, 10 D-glucose, and $26 \mathrm{NaHCO}_{3}$, bubbled with $95 \% \mathrm{O}_{2} / 5 \% \mathrm{CO}_{2}$. Slices were incubated in the chamber for $60-90 \mathrm{~min}$ before recording. For experiments in which both field and whole-cell recordings were obtained, slices were maintained in a submersion chamber as described below.

Extracellular field potentials were recorded through glass microelectrodes filled with ACSF (1-2 M $\Omega$ ) using a model 1800 A-M Systems AC amplifier and were acquired on a personal computer (PC) using the Scope software package (gift from Dr. G. Rose) or pCLAMP6 (Axon Instruments). Data were analyzed off-line using Scope, pCLAMP6, or Igor Pro (Wavemetrics). Synaptic field potentials were elicited by electrical stimulation of Schaffer collateral afferent axons. A bipolar tungsten electrode (Fred Haer) was used to apply constant-current electrical pulses $(225-450 \mu \mathrm{A} ; 0.1 \mathrm{msec}$ pulses at 1 per $30 \mathrm{sec}$ or 1 per min). Recording electrodes were placed in the stratum pyramidale or stratum radiatum at a depth of $75-100 \mu \mathrm{m}$ and $\sim 200 \mu \mathrm{m}$ from the stimulating electrode.

For long-term potentiation (LTP) experiments, hippocampal slices from $\mathrm{P} 18$ to $\mathrm{P} 20$ rats were maintained and recorded in ACSF that contained (in mM): $116 \mathrm{NaCl}, 3.5-5.37 \mathrm{KCl}, 1.02 \mathrm{NaH}_{2} \mathrm{PO}_{4}, 3.2 \mathrm{CaCl}_{2}$, $0.83 \mathrm{MgSO}_{4}, 10$ D-glucose, and $26.2 \mathrm{NaHCO}_{3}$, bubbled with $95 \% \mathrm{O}_{2} / 5 \%$ $\mathrm{CO}_{2}$. For half of these experiments, pyrroloquinoline quinone (PQQ; $200 \mu \mathrm{M}$ ) was added to the ACSF 30-50 min before and throughout recording from area CA1. Input and output curves were obtained, and the stimulus intensity that elicited $50 \%$ of the maximum EPSP slope was used for both test and tetanic stimulation. Test stimuli were applied once every $30 \mathrm{sec}$ for $10 \mathrm{~min}$ before the first tetanus to confirm the stability of baseline responses. The tetani consisted of two 1 sec epochs of $100 \mathrm{~Hz}$ pulses (100 $\mu \mathrm{sec}$ pulse duration) applied $20 \mathrm{sec}$ apart.

Whole-cell recording. For whole-cell recording experiments, 300-400 $\mu \mathrm{m}$ hippocampal slices were prepared from P10 to P18 rats as described above or using a vibratome. Individual slices were incubated for $1 \mathrm{hr}$ before being transferred to a custom-modified submersion chamber (Warner Instruments) for recording. ACSF (in mM, $119 \mathrm{NaCl}, 2.5 \mathrm{KCl}$, $1 \mathrm{NaH}_{2} \mathrm{PO}_{4}, 2.5 \mathrm{CaCl}_{2}, 1.3 \mathrm{MgSO}_{4}, 11 \mathrm{D}$-glucose, $26.2 \mathrm{NaHCO}_{3}$, and 10-30 sucrose, bubbled with $95 \% \mathrm{O}_{2} / 5 \% \mathrm{CO}_{2}$ ) was superfused at a flow rate of $60-80 \mathrm{ml} / \mathrm{hr}$ at room temperature. Glycine was not added to the recording media to maintain conditions similar to those used for field recordings. For NMDAR-mediated EPSC (nEPSC) recordings, slices were maintained at $32 \pm 1^{\circ} \mathrm{C}$ because this was necessary to achieve ictal field activity.

Whole-cell voltage-clamp recordings were obtained from neurons in st. pyramidale of area CA1. Gigaohm seals were formed on pyramidalshaped neurons under visual guidance using infrared differential interference contrast video microscopy (Stuart et al., 1993) or on neurons in st. pyramidale using the "blind"-patch technique (Blanton et al., 1989). Neurons were visualized using a Nikon Optiphot microscope with a Hitachi KP-116 camera and Panasonic video monitor. Recordings were obtained using an Axopatch 200A amplifier (Axon Instruments), and data were digitized and stored on a PC using Clampex (pCLAMP6; Axon Instruments), which also was used to generate command voltage protocols.

The patch pipette solution consisted of (in mM): 123 Cs-gluconate, 4 $\mathrm{NaCl}, 2 \mathrm{MgCl}_{2}, 10$ EGTA, 10 HEPES, 2 Na-ATP, and 0.3 GTP, pH 7.2, or a solution of the same composition except with Cs-gluconate at 103 $\mathrm{mm}$ and $20 \mathrm{~mm}$ tetraethylammonium chloride added. Results were similar with either pipette solution. Electrodes had resistances of 4-7 M $\Omega$ when filled, cell-attached seals were $>5 \mathrm{G} \Omega$, and uncompensated series resistances in the whole-cell configuration were typically 10-15 $\mathrm{M} \Omega$ (range, 6-27 M $\Omega$ ). Series resistance $\left(R_{s}\right)$ was monitored throughout experiments by applying brief $-5 \mathrm{mV}$ voltage steps before and after each stimulus. Cells were rejected if $R_{s}$ was unstable or increased to $>34 \mathrm{M} \Omega$. Apparent input resistances ranged from $600 \mathrm{M} \Omega$ to $\gg 1 \mathrm{G} \Omega$. Data were analyzed off-line using Clampfit (pCLAMP6; Axon Instruments) or on a Macintosh computer using Axograph 3.0 (Axon) and Igor Pro (Wavemetrics).

For recording NMDA-evoked currents, tetrodotoxin $(0.5-1 \mu \mathrm{M})$ was added to the ACSF, and cells were held at 0 or $+10 \mathrm{mV}$ between protocols to inactivate voltage-gated $\mathrm{Na}^{+}$and $\mathrm{Ca}^{2+}$ channels. NMDA was applied by focal pressure ejection from a patch pipette (1 mM NMDA dissolved in ACSF) using a Picospritzer (General Valve, Fairfield, NJ) or by $10-12 \mathrm{sec}$ superfusion of $100 \mu \mathrm{M}$ NMDA. When superfused, the NMDA concentration peaked more slowly than did the superfusion time because of the slow fluid exchange in our gravity perfusion system. Therefore, voltage ramps $(12 \mathrm{mV} / \mathrm{sec})$ were applied every $20 \mathrm{sec}$ starting from a fixed time relative to NMDA superfusion, and control ramp responses were subtracted from the peak ramp responses to obtain $I-V$ curves. $I-V$ curves obtained in this manner were reliably reproducible. The same protocols were used to examine AMPA/kainate receptor function, except that $500 \mu \mathrm{M}$ AMPA was applied by focal pressure ejection or $25 \mu \mathrm{M}$ kainate was applied by superfusion.

EPSCs were evoked by electrical stimulation of Schaffer collateral afferent axons as described for field potentials, except that pulses of 20-200 $\mu \mathrm{A}$ intensity were used. nEPSCs were isolated by the addition of $20-40 \mu \mathrm{M}$ CNQX and $20-100 \mu \mathrm{M}$ picrotoxin or $40 \mu \mathrm{M}$ bicuculline methiodide to the ACSF. Non-NMDAR-mediated EPSCs were recorded in the presence of $100 \mu \mathrm{M}$ picrotoxin and $50 \mu \mathrm{M}$ D,L-2-amino-5phosphonopentanoic acid (AP-5). To examine the effects of ictal activity on NMDAR redox state, slices were first exposed to $\mathrm{Mg}^{2+}$-free ACSF until spontaneous ictal activity had been observed for $30 \mathrm{~min}$. Then, control $\left(\mathrm{Mg}^{2+}\right.$-containing) ACSF was washed in, and nEPSCs were recorded beginning 30-60 min after the cessation of ictal activity.

In vivo chemical convulsant testing. Rat pups at postnatal day 12 were maintained at $33-34^{\circ} \mathrm{C}$. PQQ $\left(20 \mathrm{mg} / \mathrm{kg} ; 2.3 \mathrm{mg} / \mathrm{ml}\right.$ in $\left.2 \% \mathrm{HCO}_{3}\right)$ or vehicle $\left(2 \% \mathrm{HCO}_{3}\right)$ was administered intraperitoneally $30 \mathrm{~min}$ before the administration of either pentylenetetrazol $(80 \mathrm{mg} / \mathrm{kg}$, i.p.) or bicuculline methiodide ( $2 \mathrm{mg} / \mathrm{kg}$, s.c.). The latency to the first myoclonic jerk, the latency to generalized convulsion, and the duration of generalized convulsion were measured for each rat by an investigator blind to the treatment group. Student's $t$ test was used to compare control and PQQ-treated groups.

Chemicals. Pentylenetetrazol (PTZ), BMI, picrotoxin, AP-5, NMDA, tetrodotoxin, tetraethylammonium chloride, DTT, 5,5'-dithio-bis(2- 
A

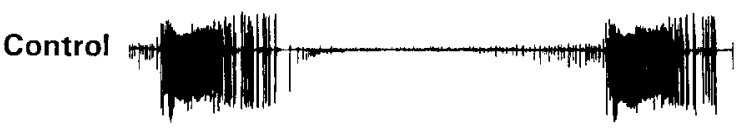

POO

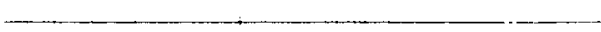

B

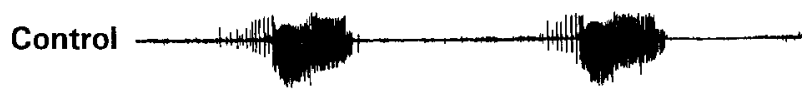

DTNB

Wash

DTT



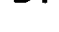

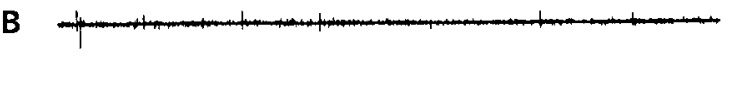

Wash

DTT

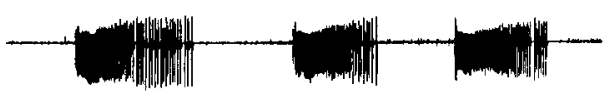

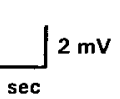

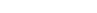

$40 \mathrm{sec}$

\begin{abstract}
Figure 1. Suppression of spontaneous ictal bursts by PQQ and DTNB. A, B, The top traces show spontaneous ictal bursts recorded from area CA1 after $30 \mathrm{~min}$ of exposure to ACSF that contained no added $\mathrm{Mg}^{2+}$. Superfusion of $200 \mu \mathrm{M}$ PQQ $(A)$ or 1 mM DTNB $(B)$ suppressed spontaneous bursting (second traces from top), and this effect persisted after washout of each agent (third traces from top). Subsequent addition of 0.5 mM DTT caused spontaneous bursts to reappear (bottom traces). In contrast to PQQ- or DTNB-treated slices, untreated control slices showed spontaneous ictal bursting that typically persisted for the duration of recording (90-240 min) and never ceased spontaneously before 90 min of continuous bursting.
\end{abstract}

nitrobenzoic acid) (DTNB), superoxide dismutase (SOD), and catalase were obtained from Sigma (St. Louis, MO). CNQX was obtained from Research Biochemicals (Natick, MA). Tris(2-carboxyethyl)phosphine hydrochloride (TCEP) was obtained from Molecular Probes (Eugene, OR). PQQ was obtained from Fluka (Buchs, Switzerland).

\section{RESULTS}

\section{Redox-active agents modulate NMDAR-dependent epileptiform activity in vitro}

We first examined the ability of agents that oxidize the NMDAR redox site to suppress NMDAR-dependent electrographic seizures in the hippocampal slice. Exposure of young rat (postnatal day 10-18) hippocampal slices to an extracellular medium that contained no added $\mathrm{Mg}^{2+}$ resulted in spontaneous ictal activity, as manifest by repetitive high-frequency bursts of population spikes (Fig. 1). This ictal pattern is characteristic of this age group and dependent on NMDAR activation (Wang and Jensen, 1996). In the absence of further treatment, the low $\mathrm{Mg}^{2+}$-induced ictal activity persisted until recording was terminated (90-240 min; $n=8)$. In contrast, the spontaneous ictal activity was completely abolished when the redox cycling agent PQQ (200 $\mu \mathrm{M} ; 6 / 6$ slices) or the sulfhydryl oxidant DTNB ( 1 mM; $9 / 14$ slices; $p<0.005$; Fisher's exact test) was applied after $10 \mathrm{~min}$ of spontaneous activity (Fig. 1). This suppression of ictal activity endured after 30-60 min of washout of either compound, but ictal firing was restored after application of the disulfide reducing agents DTT (0.5-1 mM; Fig. 1) or TCEP (500 $\mu \mathrm{M}$; data not shown). Notably, when DTT was applied to slices exhibiting low $\mathrm{Mg}^{2+}$-induced ictal activity without previous exposure to DTNB or PQQ, the interval between ictal events gradually decreased and within 10 min evolved into continuous spiking that lasted several minutes before all spontaneous activity gradually disappeared $(n=5$; data not shown).

In some experiments, epileptiform CA1 field potentials were evoked by Schaffer collateral stimulation in $\mathrm{Mg}^{2+}$-free medium. Consistent with their effects on spontaneous bursting, DTNB or PQQ abolished the late spikes in the evoked epileptiform responses, and the late spikes were restored only by subsequent exposure to DTT (Fig. 2). DTNB or PQQ, however, did not inhibit the initial population spike. Taken together, these data indicated that agents that oxidize the NMDAR redox site suppress NMDAR-dependent epileptiform activity in vitro without apparent effects on non-NMDAR-mediated synaptic transmission.

\section{Redox modulation of bicuculline-induced epileptiform responses}

To investigate further the specific contribution of NMDAR modulation in the modification of seizure activity by redox agents, we examined the effects of these agents on evoked epileptiform activity induced by the $\mathrm{GABA}_{\mathrm{A}}$ receptor inhibitor BMI, a model that is less dependent on NMDAR activation. Slices were exposed to a low concentration of BMI $(4-7 \mu \mathrm{M})$, because this allowed synaptically evoked epileptiform responses to be recorded without spontaneous activity. Under this condition, the evoked responses exhibited multiple population spikes as in the low- $\mathrm{Mg}^{2+}$ condition (Fig. $3 A$ ). In contrast to the low- $\mathrm{Mg}^{2+}$ condition, application of DTNB and PQQ had no effect on the late spikes without previous addition of a reducing agent but completely reversed the effects of reducing agents. Figure $3 A$ shows that DTT $(500 \mu \mathrm{M})$ significantly increased the numbers of spikes in the evoked responses and that this increase persisted during DTT washout. The numbers of spikes were decreased by subse- 

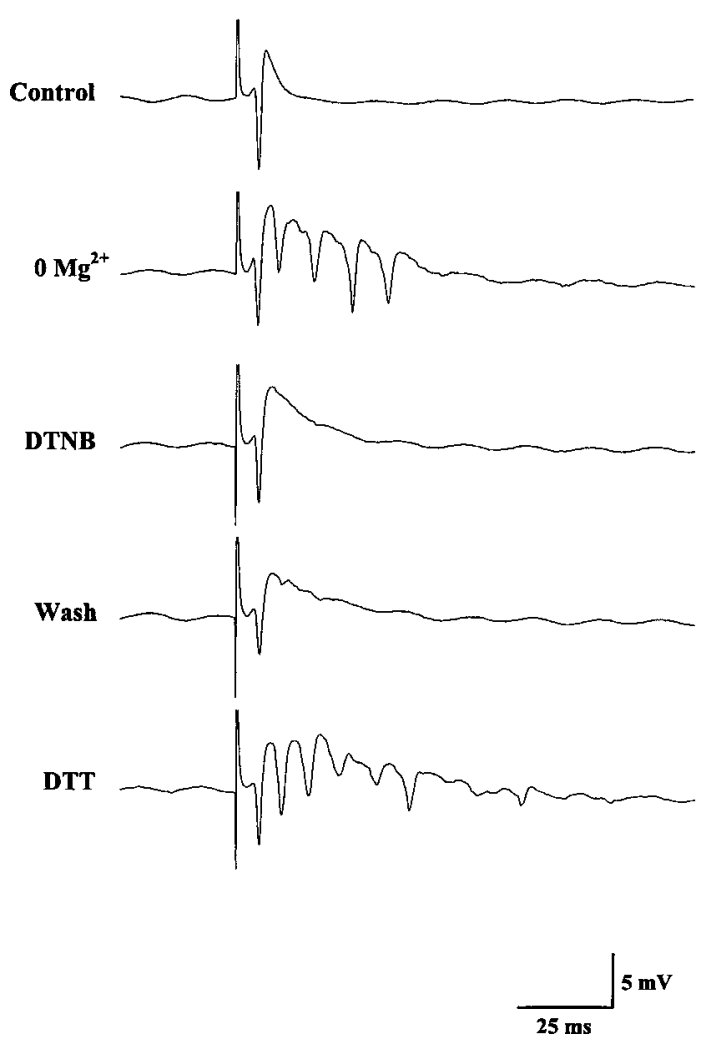

Figure 2. Inhibition of evoked epileptiform field potentials in $\mathrm{Mg}^{2+}$-free ACSF by PQQ and DTNB. The data traces show the emergence of multiple peaks in the evoked response during exposure to $\mathrm{Mg}^{2+}$-free media and the inhibition of these later peaks by subsequent addition of 1 mM DTNB to the bath. As with the spontaneous ictal bursts, the evoked late peaks were not observed during DTNB washout but were restored by DTT application.

quent exposure to PQQ $(100 \mu \mathrm{M})$ or DTNB $(500 \mu \mathrm{M}$; data not shown), and this decrease also persisted after washout of either compound. As indicated in the bar graphs in Figure $3 C$, the number of spikes in the evoked response significantly increased within $10 \mathrm{~min}$ of exposure to DTT from $4.8 \pm 0.7$ to $9.6 \pm 2.3$ peaks (mean $\pm \mathrm{SEM} ; n=5 ; p<0.05$; one-way repeated measures ANOVA using Student-Newman-Keuls post hoc pairwise comparison) and remained increased after $30 \mathrm{~min}$ of washout of DTT (12.2 \pm 2.9 peaks). Subsequent application of $100 \mu \mathrm{M}$ PQQ decreased the number of spikes in the evoked response within 10 $\min (5.6 \pm 0.8$ peaks; significantly different from DTT, $p<0.05)$, and this effect also was unchanged after $30 \mathrm{~min}$ of washout (5.4 \pm 0.8 peaks). Notably, although PQQ and DTNB rapidly and persistently reversed the DTT effect, they did not decrease the numbers of spikes beyond pre-DTT baseline levels and did not alter the evoked responses without previous DTT application. This was in contrast to their effects on low $\mathrm{Mg}^{2+}$-induced epileptiform activity and suggested that NMDARs did not contribute significantly to evoked epileptiform responses in the presence of BMI at a concentration that was below the threshold to elicit spontaneous ictal activity.

To determine more directly whether NMDAR modulation by redox agents had mediated their effects on epileptiform responses, we repeated this experiment with NMDARs blocked by the specific antagonist AP-5 $(100 \mu \mathrm{M})$. As with DTNB and PQQ, AP-5 had no effect on the compound evoked response induced by partial $\mathrm{GABA}_{\mathrm{A}}$ receptor blockade with $\mathrm{BMI}$, indicating that this epileptiform response was not NMDAR dependent. In the presence of AP-5, the numbers of spikes in the evoked responses were completely unchanged by each of the redox agents (Fig. $3 B, D ; n=$ 4). This suggested that NMDAR-independent epileptiform activity was insensitive to modulation by redox agents and that, in the absence of AP-5, DTT had caused the emergence of an NMDAR-mediated component of epileptiform activity that was subsequently suppressed by PQQ and DTNB.

Under certain conditions, PQQ may promote the formation of oxygen-derived free radicals (Paz et al., 1992; Fluckiger et al., 1993), which also oxidize the NMDAR redox site (Aizenman et al., 1990; Aizenman, 1995). To exclude the possibility that the effects of PQQ were mediated indirectly via the generation of free radicals, we repeated this experiment in slices continuously exposed to the enzymatic free-radical scavengers SOD (100 units/ $\mathrm{ml}$ ) and catalase (CAT; 210 units $/ \mathrm{ml})$. As indicated in the bar graphs in Figure $3 E$, the results obtained in the presence of SOD and CAT were similar to those obtained in their absence, and no significant differences were found between the same groups compared with versus without SOD and CAT. Thus, the generation of extracellular free radicals did not contribute to the modulation of epileptiform activity by PQQ.

It is noteworthy that, in addition to prolonging the evoked responses in BMI, DTT caused the emergence of spontaneous synchronous field activity that persisted during DTT washout but was completely abolished by subsequent application of DTNB or PQQ (data not shown). AP-5 also prevented this DTT-induced spontaneous activity, further indicating that the induction of synchronous seizure-like activity was NMDAR dependent. Thus, our data suggested that, under a proconvulsant condition, the mass reduction of NMDAR redox sites may be sufficient to precipitate spontaneous epileptiform activity.

\section{Effects of redox reagents on NMDAR-mediated whole- cell currents in CA1 neurons}

Having established effects of redox-active compounds on epileptiform activity in hippocampal slices, we next obtained whole-cell voltage-clamp recordings from neurons in the pyramidal cell layer of area CA1 to determine whether these redox reagents directly modulated NMDAR function at concentrations that altered epileptiform field activity. As shown in Figure 4, NMDA-evoked currents were potentiated by TCEP $(200-400 \mu \mathrm{M})$, and this effect was rapidly and persistently reversed by subsequent exposure to PQQ $(200 \mu \mathrm{M})$ or DTNB $(500 \mu \mathrm{M})$. TCEP significantly increased peak NMDA-evoked currents $[167.4 \pm 15.9 \%$ of control (mean \pm $\mathrm{SEM}) ; n=8 ; p<0.05$, one-way ANOVA with post hoc group comparison], and PQQ significantly decreased the NMDAevoked current compared with post-TCEP treatment $(95.9 \pm$ $5.5 \%$ of control; $n=7 ; p<0.05)$. Additionally, each of these agents exerted modulation with no effect on the voltage dependence or reversal potential of NMDA-evoked currents (Fig. $4 B, C)$. As with field recordings, the effect of TCEP on NMDAevoked currents was mimicked by DTT (500 $\mu \mathrm{M}$; data not shown), and the effect of PQQ was mimicked by DTNB $(500 \mu \mathrm{M})$.

Figure $4 D$ illustrates the time course of the effects of redox agents on currents evoked by focal NMDA application. Clearly, the potentiation of NMDA-evoked currents by TCEP persisted after washout but was rapidly reversed by PQQ or DTNB. Similarly, no changes were observed after PQQ washout, but NMDAevoked currents could be potentiated again by exposure to TCEP. The persistence of these effects paralleled those observed with 


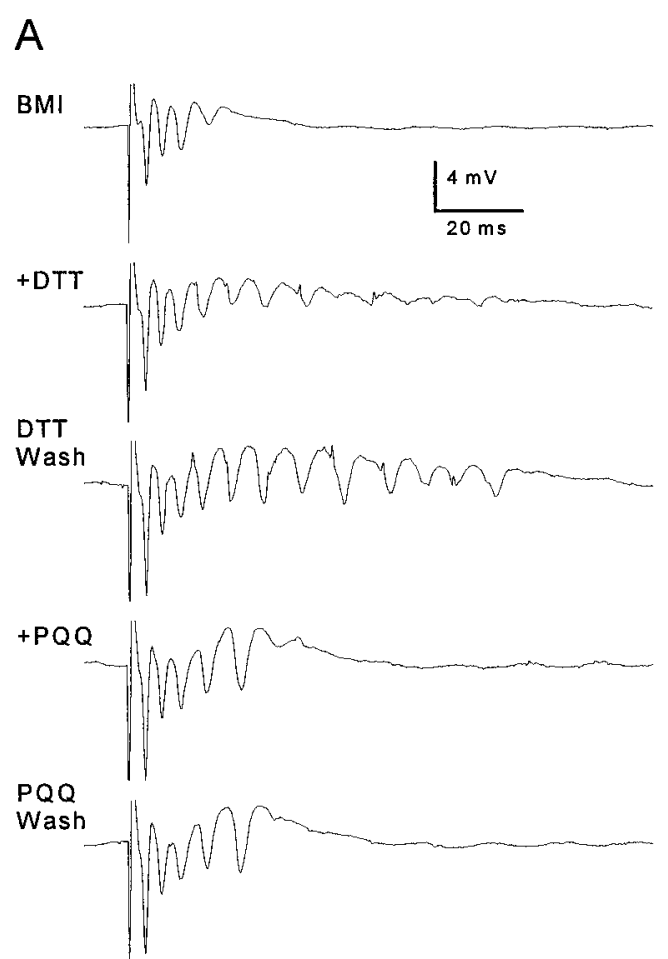

$\mathrm{C}$

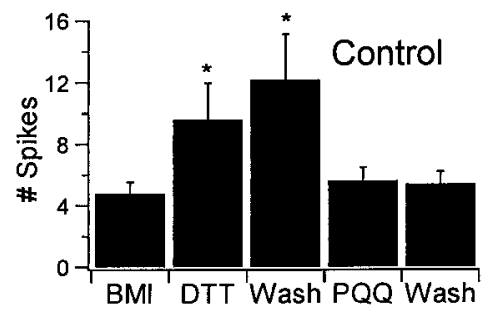

D

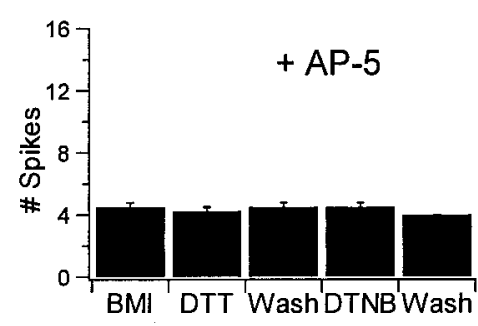

B
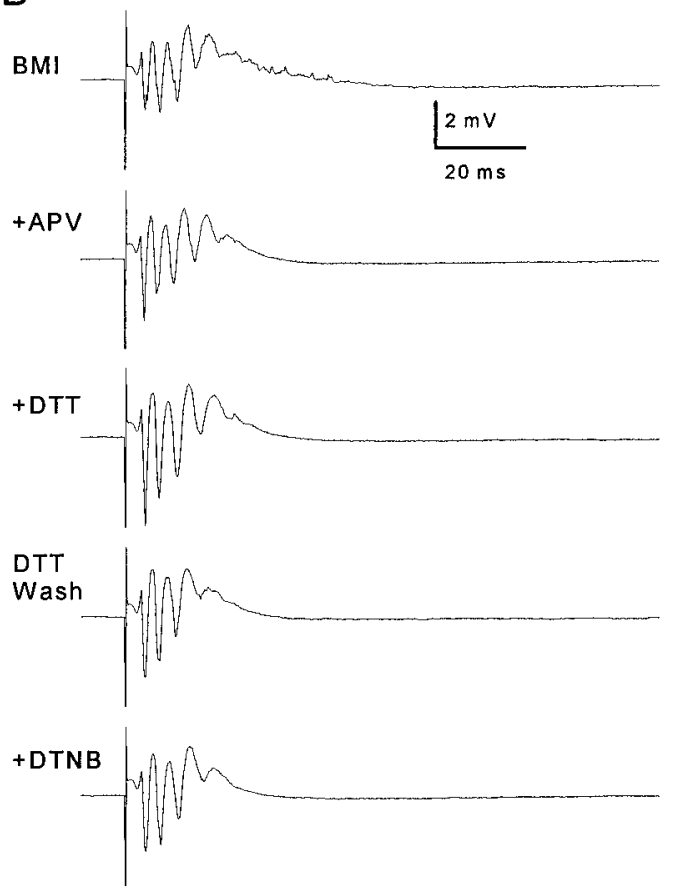

E

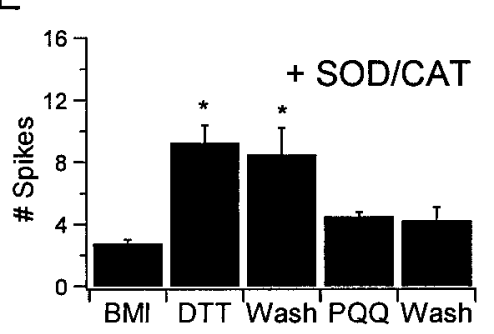

Figure 3. Redox modulation of bicuculline-induced epileptiform activity. A, The top trace shows multiple CA1 population spikes evoked by a single stimulus applied to the Schaffer collateral afferents in the presence of $7 \mu \mathrm{M}$ BMI. Application of $500 \mu \mathrm{M}$ DTT resulted in an increased number of population spikes in the evoked response (second trace from top), and this effect persisted after DTT washout (third trace from top). Subsequent application of $100 \mu \mathrm{M}$ PQQ reduced the number of spikes to the pre-DTT baseline level ( fourth trace from top), and this effect also persisted after PQQ washout (bottom trace). B, Similar recordings obtained in the presence of $100 \mu \mathrm{M}$ AP-5 (APV) showed no modulation by redox agents when NMDARs were blocked. $C$, The bar graphs summarize the effects of DTT and PQQ on evoked CA1 field potentials in low BMI. DTT (0.5 mM) significantly increased the number of population spikes in the evoked response, and this increase persisted after DTT washout. The effect of DTT was completely reversed by PQQ $(100 \mu \mathrm{M})$, but the number of spikes in the evoked response did not decrease beyond pre-DTT levels. $D$, The bar graphs illustrate the lack of redox modulation of epileptiform responses in the presence of $100 \mu \mathrm{M}$ AP-5 $(n=4)$. E. Identical experiments in the presence of SOD (100 units $/ \mathrm{ml}$ ) and CAT (210 units/ml) showed no differences from those obtained in the absence of SOD and CAT $(n=4)$, indicating that the effects of PQQ were not mediated by the generation of free radicals.

field recordings and was consistent with modulation that is mediated by the modification of a covalent bond. The persistence of potentiation after TCEP washout also indicated that the TCEPinduced potentiation was not mediated by zinc chelation, because this effect would be expected to reverse rapidly after washout (Paoletti et al., 1997). Additionally, PQQ and TCEP had no effect on pharmacologically isolated AMPA/kainate receptor-mediated currents $(n=4$; data not shown), consistent with previous work that showed no effect of DTNB and DTT on AMPA/kainate receptors (Aizenman et al., 1989).

Whereas DTNB and PQQ powerfully inhibited NMDAevoked responses that were potentiated by reducing agents, they did not significantly inhibit responses beyond baseline values before the addition of a reductant (Fig. $4 B, D$ ). This was a consistent observation regardless of which compound was applied first and indicated that NMDAR redox sites were mostly oxidized under baseline (nonepileptogenic) conditions in our slice preparation. These data were consistent with field recordings obtained with partial $\mathrm{GABA}_{\mathrm{A}}$ receptor blockade but were apparently inconsistent with the powerful suppression of low $\mathrm{Mg}^{2+}$-induced ictal activity by DTNB and PQQ. However, these data could be reconciled if NMDAR redox sites became reduced by endogenous factors under the $\mathrm{Mg}^{2+}$-free epileptogenic condition.

\section{Seizure activity induces endogenous redox potentiation of NMDARs}

To investigate whether epileptiform activity may cause potentiation of NMDARs by endogenous redox-active factors, we examined the sensitivity of pharmacologically isolated nEPSCs to inhibition by DTNB immediately after the termination of low $\mathrm{Mg}^{2+}$-induced ictal activity. Because the inhibition of NMDARmediated responses by oxidizing agents was increased after pre- 
Figure 4. NMDA-evoked whole-cell currents are modulated by redox reagents at concentrations that modulated epileptiform activity. $A, I-V$ curves for NMDA-evoked currents were obtained by subtracting the responses to command voltage ramps from -90 to +30 $\mathrm{mV}$ before and during brief NMDA superfusion. Subtracted current responses revealed characteristic voltagedependent currents that reversed between 0 and $+10 \mathrm{mV}$, and the superimposed subtracted ramps for three consecutive NMDA applications show the reproducibility of responses obtained in this manner. $B$, Superimposed $I-V$ curves show that baseline NMDAevoked currents (trace 1) were significantly potentiated by exposure to 500 $\mu \mathrm{M}$ TCEP (trace 2) and subsequently diminished by $200 \mu \mathrm{M}$ PQQ (trace 3). Each trace is an average of three subtracted ramp responses. $C$, Ramp currents from $B$ scaled to the largest current at $-20 \mathrm{mV}$ showed that TCEP and PQQ exerted modulation with no effect on the voltage dependence or reversal potential of the NMDA-evoked currents. $D$, Persistence of the effects of redox reagents on currents evoked by focal NMDA application are shown. The graph shows the peak amplitude of individual current responses to focal NMDA application (holding potential $=+30 \mathrm{mV}$ ) as a function of time for a CA1 pyramidal neuron that was recorded for $3 \mathrm{hr}$. (Horizontal bars show the time of superfusion of each redox agent.) The initial application of $400 \mu \mathrm{M}$ TCEP caused a gradual increase in the peak NMDAevoked current that remained unchanged during TCEP washout but very rapidly returned to baseline after subsequent exposure to $200 \mu \mathrm{M}$ PQQ. Responses were potentiated again by a second TCEP application, and this effect was reversed by exposure to $500 \mu \mathrm{M}$ DTNB, mimicking the effect of PQQ. Individual traces are shown at the top for the points labeled in the graph. Calibration: vertical, $100 \mathrm{pA}$; horizontal, $10 \mathrm{sec}$.

Figure 5. NMDAR-mediated synaptic currents were more sensitive to inhibition by DTNB after ictal activity. $A$, Shown superimposed are averaged (4-6 events) NMDAR-mediated EPSCs recorded at a holding potential of $-15 \mathrm{mV}$ in a CA1 neuron from a control slice before and after exposure to $500 \mu \mathrm{M}$ DTNB. $B$, The same is shown for a neuron recorded from a slice that had been exposed to $\mathrm{Mg}^{2+}$. free media and exhibited spontaneous field activity for $30 \mathrm{~min}$ before the initiation of whole-cell recording (see Materials and Methods). $C$, The bar



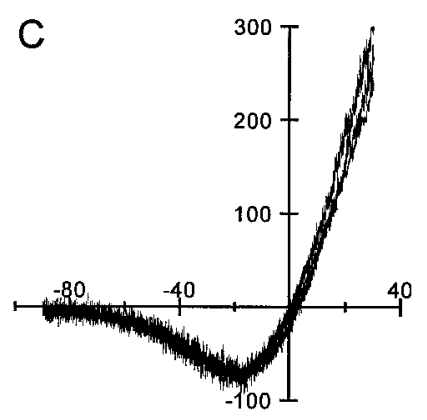
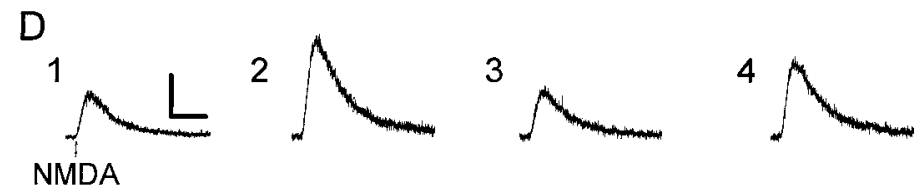

5

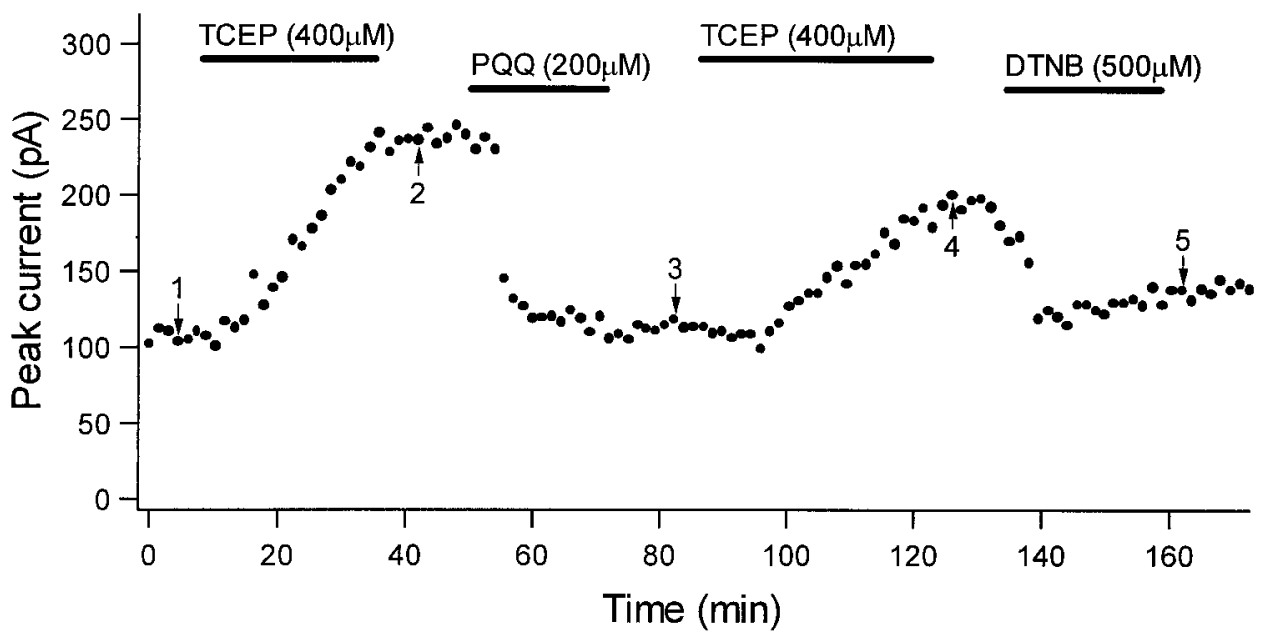
graphs show summary data. The increased sensitivity of NMDAR-mediated EPSCs to DTNB-induced inhibition indicated that the epileptogenic condition had caused NMDAR redox sites to be reduced by endogenous factors.

vious exposure to reducing agents, we reasoned that nEPSCs in slices in which mass reduction of NMDAR redox sites had occurred should exhibit significantly greater inhibition by an oxidizing agent compared with that in control slices. In slices returned to control medium after exhibiting low $\mathrm{Mg}^{2+}$-induced ictal activity for $30 \mathrm{~min}$, DTNB $(500 \mu \mathrm{M})$ exerted significantly greater inhibition of nEPSCs $(45.8 \pm 0.85 \% ; n=4)$ compared with that in slices maintained for the same duration in control medium
(17.8 $\pm 6.1 \% ; n=4 ; p<0.0004$; Fig. 5). Additionally, the increased sensitivity of nEPSCs to DTNB was observed as long as $1 \mathrm{hr}$ after the termination of ictal activity by returning slices to control medium. These data strongly suggested that low $\mathrm{Mg}^{2+}$ induced epileptiform activity had caused the reduction of NMDAR redox sites that resulted in potentiation of NMDAR function even after the cessation of ictal activity. Because no reducing agents were added, these data implicated endogenous 

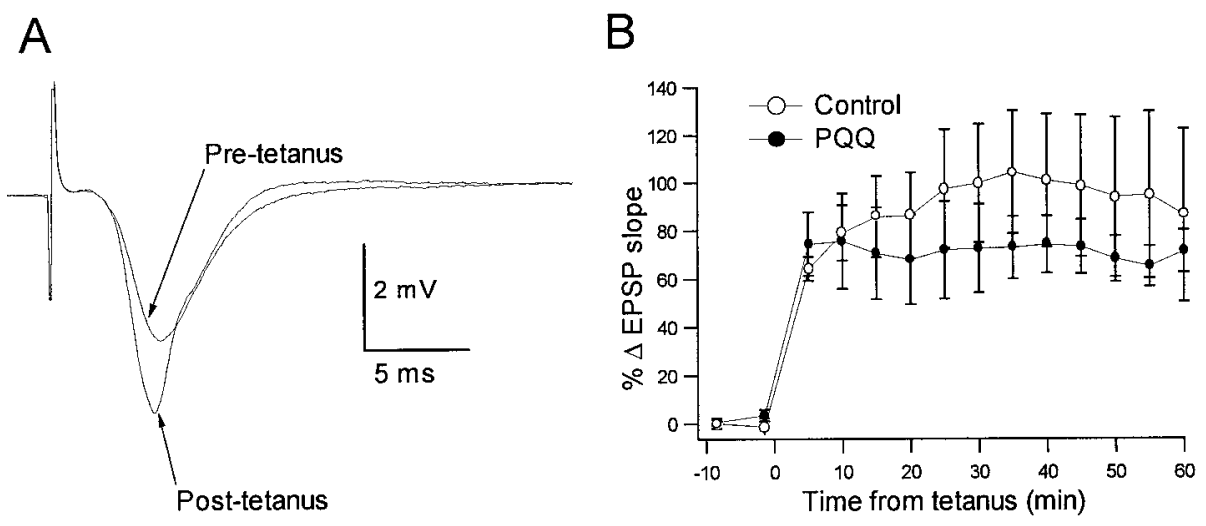

Figure 6. Anticonvulsant concentrations of PQQ do not interfere with NMDAR-dependent synaptic plasticity. LTP of the CA1 field EPSP evoked by Schaffer collateral stimulation was induced by tetanic stimulation in control slices $(n=4)$ and in slices maintained in $200 \mu \mathrm{M}$ PQQ $(n=4)$ for $30 \mathrm{~min}$ before tetanus and for the duration of recording. $A$, The superimposed traces show averaged field EPSPs ( 5 events) before and after tetanus in a representative slice recorded in the presence of PQQ. $B$, The summary graph shows the mean increase in EPSP slope as a function of time from the tetanus for the two conditions. The magnitude of LTP was not statistically different in the presence versus the absence of PQQ (two-way repeated measures ANOVA with post hoc comparison).
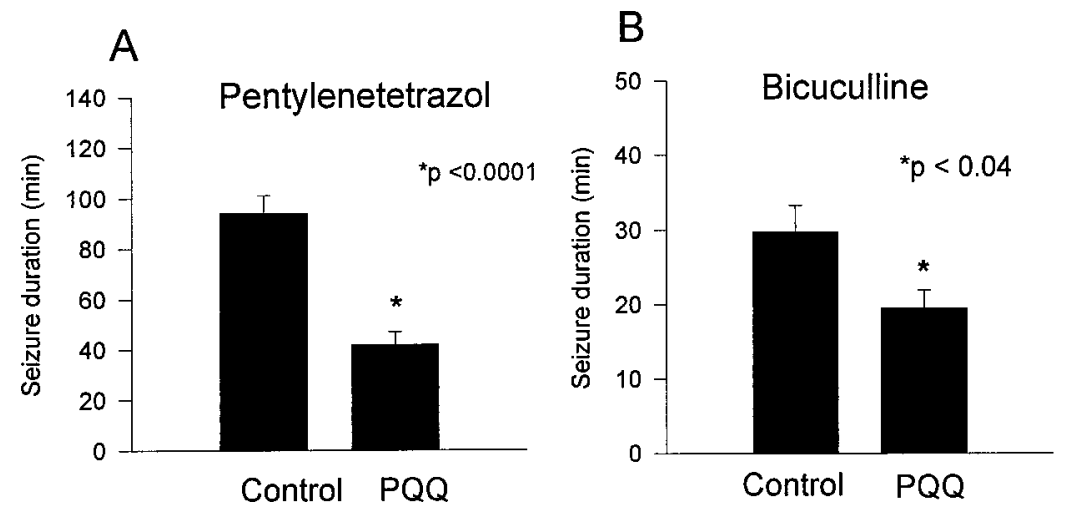

Figure 7. Systemic pretreatment with PQQ significantly attenuated seizures induced by PTZ or BMI. $A, B$, The bar graphs show the mean duration of PTZ-induced $(A)$ and BMI-induced $(B)$ seizures for control $\left(\mathrm{HCO}_{3}\right.$ vehicletreated) and PQQ-treated groups. The durations of PTZ- and BMI-induced generalized seizures were significantly decreased in PQQ-treated animals. reducing compounds, generated in brain tissue during the seizure activity, as mediators of this effect.

\section{Oxidation of NMDAR redox sites is permissive of long-term potentiation}

In contrast to many experimental NMDAR antagonists, our data showed little effect of PQQ or DTNB on baseline NMDAR function. Thus, these agents appeared to achieve inhibition of epileptiform activity via the reversal of pathological potentiation of NMDAR function rather than simply via downmodulation of physiological NMDAR function. This suggested that therapeutic use of this class of agents might be without significant adverse effects on NMDAR-dependent physiological function, such as synaptic plasticity. To examine this hypothesis, we compared hippocampal LTP in the presence and absence of PQQ. LTP is an extensively used model of NMDAR-dependent synaptic plasticity thought to underlie learning and memory (Cain, 1997). As shown in Figure $6 A$, tetanic stimulation of Schaffer collateral axons induced long-lasting potentiation of the slope of the field EPSP recorded from area CA1 st. radiatum. In slices continuously exposed to PQQ $(200 \mu \mathrm{M})$ beginning $30 \mathrm{~min}$ before tetanic stimulation, the magnitude of LTP was not significantly different from that achieved in control slices (Fig. 6B). This finding indicated that, unlike most pharmacological NMDAR antagonists, PQQ might inhibit pathological NMDAR potentiation without interfering with NMDAR-dependent physiological processes.

\section{Anticonvulsant effect of a systemically administered redox agent in vivo}

Unlike most redox-active agents used in vitro, $\mathrm{PQQ}$ is systemically tolerated and distributed in brain after systemic administration (Smidt et al., 1991). We therefore were able to examine the anticonvulsant efficacy of PQQ against chemoconvulsant-induced seizures in vivo. Behavioral seizures were induced in rat pups by either PTZ or BMI, because these are well characterized chemoconvulsants that induce seizures that are inhibited by NMDAR antagonists (Dingledine et al., 1990). Systemic pretreatment with PQQ (20 mg/kg) caused no obvious behavioral changes but significantly attenuated status epilepticus induced by PTZ (80 $\mathrm{mg} / \mathrm{kg}$, i.p.) or BMI (2 mg/kg, s.c.) (Fig. 7). The duration of PTZ-induced generalized seizure was significantly decreased in PQQ-treated animals $(41.9 \pm 5.3 \mathrm{~min} ; n=10)$ compared with vehicle-treated controls $(94.2 \pm 7.1 \mathrm{~min} ; n=10 ; p<0.001)$. PQQ pretreatment also significantly increased the latency to PTZinduced myoclonic jerks (109.9 \pm 52.9 and $62.9 \pm 8.4 \mathrm{sec}$ for PQQ- and vehicle-treated groups, respectively; $p<0.007)$ and the latency to generalized seizure $(375.9 \pm 72.3$ and $197.3 \pm 37.37 \mathrm{sec}$ for PQQ- and vehicle-treated groups, respectively; $p<0.001)$. The duration of BMI-induced seizures also was significantly decreased by PQQ pretreatment [19.5 \pm 2.4 min for PQQ-treated $(n=18)$ vs $29.7 \pm 3.6$ min for vehicle-treated $(n=19) ; p<0.03$ ], although significant differences were not observed in the latencies to myoclonic jerks (vehicle, $117.4 \pm 11.9 \mathrm{sec}$; PQQ, $118.7 \pm 11$ sec) or generalized seizure (vehicle, $179.1 \pm 10.6 \mathrm{sec}$; PQQ, $201.9 \pm 21 \mathrm{sec}$ ). These data demonstrated, in two established NMDAR-dependent in vivo seizure models, that behavioral seizures were inhibited by systemic administration of an agent that is capable of oxidizing the NMDAR redox site.

\section{DISCUSSION}

Our data indicate that the NMDAR redox modulatory site has a previously unidentified critical role in the expression of seizure activity. We observed that agents that oxidize the NMDAR redox site significantly inhibited epileptiform activity in normal rat hippocampal slices exposed to epileptogenic conditions, 
whereas reducing agents induced opposite effects. The absence of these effects in the presence of the NMDAR antagonist AP-5 supported the hypothesis that the modulation epileptiform activity by redox-active agents was mediated by their modulation of NMDAR function. Whole-cell voltage-clamp recordings demonstrated that DTNB and PQQ oxidized the redox sites of native NMDARs in situ at concentrations that inhibited epileptiform activity yet had little effect on NMDAR function under baseline (nonepileptogenic) conditions. In sharp contrast, DTNB strongly inhibited NMDAR-mediated synaptic currents in slices that had exhibited previously low $\mathrm{Mg}^{2+}$-induced ictal activity. This indicated that NMDAR redox sites that are mostly oxidized under baseline conditions can be reduced by endogenous factors under epileptogenic conditions.

Evidence of endogenous potentiation of NMDAR function by redox-active compounds during ictal activity in vitro may be relevant to seizures in vivo, because prolonged seizures and status epilepticus are associated with shifts in redox state in brain tissue (Tenny et al., 1980; Dora, 1983; Fujikawa et al., 1988). Similar redox potentiation of NMDAR function has been observed in mature rat hippocampal slices in response to energy depletion induced by brief anoxia/aglycemia ("anoxic LTP") (Gozlan et al., 1994). Thus, evidence indicates that disturbances of brain metabolism can induce pathological cellular redox changes that cause increased NMDAR activity and, hence, synaptic hyperexcitability. Mass reduction of NMDAR redox sites during seizures may create a pathological state of enhanced NMDAR function that could be targeted by pharmacological agents. In agreement with this notion, we observed that systemic administration of PQQ significantly decreased the duration of chemoconvulsant-induced seizures in vivo. Agents that specifically target the NMDAR redox site or that maintain homeostasis of the extracellular redox environment may therefore have therapeutic value in the treatment of seizures and status epilepticus.

It is noteworthy that whereas reducing agents enhance excitatory field potentials and NMDAR-dependent tetanic LTP in hippocampal slices (Tolliver and Pellmar, 1987; Tauck and Ashbeck, 1990), the oxidizing agent DTNB reverses these effects without blocking LTP (Tauck and Ashbeck, 1990; Gozlan et al., 1995; Bernard et al., 1997). Here, we showed that concentrations of PQQ that inhibited epileptiform activity also did not significantly inhibit NMDAR-dependent tetanic LTP. In agreement with this, we reported previously that neuroprotective doses of PQQ produced no alterations in the spectral EEG or in behavior in neonatal rats (Jensen et al., 1994). Our findings indicate that the development of agents that efficiently and specifically oxidize the NMDAR redox site could provide a means to reverse pathological NMDAR potentiation without inhibiting NMDARmediated physiological function.

\section{Specificity of redox agents}

Our data are consistent with a specific action of DTNB and PQQ at the NMDAR redox site in vitro and in vivo, yet it is possible that other effects contributed to the suppression of seizure activity. PQQ is a redox cycling agent and could have altered synaptic transmission or seizure activity indirectly via the generation or scavenging of free radicals, which can modulate NMDARs (Aizenman et al., 1990; Aizenman, 1995) and are neurotoxic (Fagni et al., 1994; Bains and Shaw, 1997; MacDonald and Stoodley, 1998). However, we observed that the enzymatic free-radical scavengers SOD and catalase did not alter the PQQ-induced inhibition of evoked and spontaneous epileptiform activity in vitro. This also was consistent with the lack of effect of SOD and catalase on PQQ modulation of NMDARs in neuronal cultures (Aizenman et al., 1992). Thus, it is unlikely that the effects of PQQ were mediated indirectly via an interaction with free radicals.

Redox-active compounds also have been shown to affect acetylcholine receptors (Hedlund and Bartfai, 1979; Lukas and Bennett, 1980) and $\mathrm{GABA}_{\mathrm{A}}$ receptors (Allan and Baier, 1992; Pan et al., 1995), both of which may play a role in the synchronization of neuronal firing in limbic seizures. However, evidence indicates that redox modulation of other neurotransmitter receptors is unlikely to have contributed to the anticonvulsant effect of oxidizing agents. DTNB downmodulates $\mathrm{GABA}_{\mathrm{A}}$ receptors in vitro (Pan et al., 1995), and therefore, oxidation of this receptor would decrease synaptic inhibition and be expected to exacerbate rather than inhibit seizure activity. Acetylcholine receptor function is enhanced by oxidizing agents, but this also would be expected to exacerbate seizures because muscarinic agonists promote synchronization and epileptiform firing in limbic brain slices (Dickson and Alonso, 1997; Williams and Kauer, 1997; Psarropoulou and Dallaire, 1998). Additionally, we observed no effect of PQQ on AMPA/kainate receptor-mediated responses, as reported previously for DTNB (Aizenman et al., 1989). Thus, our current knowledge of the redox modulation of neurotransmitter receptors and their roles in the patterning of seizure activity is consistent with our hypothesis that the modulation of epileptiform activity by redox-active compounds was achieved via modulation of NMDAR function.

Because of the physical nature of redox modulation, it is possible that the function of many as yet undetermined elements of synaptic signaling and neuronal excitability could be modulated by redox-active compounds. Evidence suggests that NMDARs exhibit a uniquely large range of functional modulation by redoxactive compounds compared with other channel proteins (Gozlan and Ben-Ari, 1995), and our data indicated that redox modulation of NMDAR function was sufficient to modulate epileptiform activity in vitro. However, in the absence of complete characterization of the effects of redox agents on every synaptic element, we cannot exclude the possibility that the modulation of seizure activity by these agents was mediated in part by multiple effects.

\section{Summary}

The present findings indicate that redox modulation of the NMDARs occurs as an acute effect of experimentally induced seizure activity and may in part underlie the self-sustaining nature of seizures, such as in status epilepticus. Reducing equivalents are known to accumulate during status epilepticus, and our data suggest that NMDAR redox modulation may be involved in a pathological link between neuronal metabolism and neurotransmission. Because NMDARs play a critical role in the synchronization of activity and maintenance of excitation in seizures, this manner of NMDAR potentiation represents a novel mechanism by which seizures could be triggered and/or sustained under epileptogenic conditions. Because oxidation of NMDAR redox sites does not appear to block NMDAR-dependent processes such as LTP, redox modulation of NMDARs or the maintenance of extracellular redox homeostasis may be a safe and effective strategy for anticonvulsant therapy. 


\section{REFERENCES}

Aizenman E (1995) Modulation of $N$-methyl-D-aspartate receptors by hydroxyl radicals in rat cortical neurons in vitro. Neurosci Lett 189:57-59.

Aizenman E, Lipton SA, Loring RH (1989) Selective modulation of NMDA responses by reduction and oxidation. Neuron 2:1257-1263.

Aizenman E, Hartnett KA, Reynolds IJ (1990) Oxygen free radicals regulate NMDA receptor function via a redox modulatory site. Neuron 5:841-846.

Aizenman E, Hartnett KA, Zhong C, Gallop PM, Rosenberg PA (1992) Interaction of the putative essential nutrient pyrroloquinoline quinone with the $N$-methyl-D-aspartate receptor redox modulatory site. J Neurosci 12:2362-2369.

Allan AM, Baier LD (1992) Effect of thiol group modification on ion flux and ligand binding properties of the GABAA-benzodiazepine receptor chloride channel complex. Synapse 10:310-316.

Bains JS, Shaw CA (1997) Neurodegenerative disorders in humans: the role of glutathione in oxidative stress-mediated neuronal death. Brain Res Brain Res Rev 25:335-358.

Bernard CL, Hirsch JC, Khazipov R, Ben-Ari Y, Gozlan H (1997) Redox modulation of synaptic responses and plasticity in rat CA1 hippocampal neurons. Exp Brain Res 113:343-352.

Blanton MG, Loturco JJ, Kriegstein AR (1989) Whole-cell recording from neurons in slices of reptilian and mammalian cerebral cortex. J Neurosci Methods 30:203-210.

Bradford HF (1995) Glutamate, GABA, and epilepsy. Prog Neurobiol 47:477-511.

Brimecombe JC, Boeckman FA, Aizenman E (1997) Functional consequences of NR2 subunit composition in single recombinant $N$-methyl-D-aspartate receptors. Proc Natl Acad Sci USA 94:11019-11024.

Cain DP (1997) LTP, genes, and learning. Curr Opin Neurobiol $7: 235-242$.

Chapman AG (1998) Glutamate receptors in epilepsy. Prog Brain Res 116:371-383.

Dannhardt G, Kohl BK (1998) The glycine site on the NMDA receptor: structure-activity relationships and possible therapeutic applications. Curr Med Chem 5:253-263.

Dickson CT, Alonso A (1997) Muscarinic induction of synchronous population activity in the entorhinal cortex. J Neurosci 17:6729-6744.

Dingledine R, McBain CJ, McNamara JO (1990) Excitatory amino acid receptors in epilepsy. Trends Pharmacol Sci 11:334-338.

Dora E (1983) Glycolysis and epilepsy-induced changes in cerebrocortical NAD/NADH redox state. J Neurochem 41:1774-1777.

Fagni L, Lafon-Cazal M, Rondouin G, Manzoni O, Lerner-Natoli M, Bockaert J (1994) The role of free radicals in NMDA-dependent neurotoxicity. Prog Brain Res 103:381-390.

Fluckiger R, Paz MA, Mah J, Bishop A, Gallop PM (1993) Characterization of the glycine-dependent redox cycling activity in animal fluids and tissue using specific inhibitors and activators. Evidence for presence of PQQ. Biochem Biophys Res Commun 196:61-68.

Fujikawa DG, Vanucci RC, Dwyer BE, Wasterlain CG (1988) Generalized seizures deplete brain energy reserves in normoxemic newborn monkeys. Brain Res 454:51-59.

Gozlan H, Ben-Ari Y (1995) NMDA receptor redox sites: are they targets for selective neuronal protection? Trends Pharmacol Sci 16:368-374.

Gozlan H, Diabira D, Chinestra P, Ben-Ari Y (1994) Anoxic LTP is mediated by the redox modulatory site of the NMDA receptor. J Neurophysiol 72:3017-3022.

Gozlan H, Khazipov R, Ben-Ari Y (1995) Multiple forms of longterm potentiation and multiple regulatory sites of $N$-methyl-Daspartate receptors: role of the redox site. J Neurobiol 26:360-369.

Hedlund B, Bartfai T (1979) The importance of thiol and disulfide groups in agonist and antagonist binding to the muscarinic receptor. Mol Pharmacol 15:531-544.

Hoshi Y, Tamura M (1993) Dynamic changes in cerebral oxygenation in chemically induced seizures in rats: study by near-infrared spectrophotometry. Brain Res 603:215-221.

Jensen FE, Gardner GJ, Williams AP, Gallop PM, Aizenman E, Rosenberg PA (1994) The putative essential nutrient pyrroloquinoline quinone is neuroprotective in a rodent model of hypoxic/ ischemic brain injury. Neuroscience 62:399-406.

Jensen FE, Wang C, Stafstrom CE, Liu Z, Geary C, Stevens MC (1998) Acute and chronic increases in excitability in rat hippocampal slices after perinatal hypoxia in vivo. J Neurophysiol 79:73-81.

Kohr G, Eckardt S, Luddens H, Monyer H, Seeburg PH (1994) NMDA receptor channels: subunit-specific potentiation by reducing agents. Neuron 12:1031-1040.

Kornhuber J, Weller M (1997) Psychotogenicity and $N$-methyl-Daspartate receptor antagonism: implications for neuroprotective pharmacotherapy. Biol Psychiatry 41:135-144.

Layton ME, Samson FE, Pazdernik TL (1998) Kainic acid causes redox changes in cerebral cortex extracellular fluid: NMDA receptor activity increases ascorbic acid whereas seizure activity increases uric acid. Neuropharmacology 37:149-157.

Li X, Wallin C, Weber SG, Sandberg M (1999) Net efflux of cysteine, glutathione and related metabolites from rat hippocampal slices during oxygen/glucose deprivation: dependence on $\gamma$-glutamyl transpeptidase. Brain Res 815:81-88.

Lukas RJ, Bennett EL (1980) Chemical modification and reactivity of sulfhydryls and disulfides of rat brain nicotinic-like acetylcholine receptors. J Biol Chem 255:5573-5577.

MacDonald RL, Stoodley M (1998) Pathophysiology of cerebral ischemia. Neurol Med Chir (Tokyo) 38:1-11.

Pan ZH, Bahring R, Grantyn R, Lipton SA (1995) Differential modulation by sulfhydryl redox agents and glutathione of GABA- and glycine-evoked currents in rat retinal ganglion cells. J Neurosci 15:1384-1391.

Paoletti P, Ascher P, Neyton J (1997) High affinity zinc inhibition of NMDA NR1-NR2A receptors. J Neurosci 17:5711-5725.

Paz MA, Fluckiger R, Gallop PM (1992) The biomedical significance of PQQ. In: Principles and applications of quinoproteins (Davidson VL, ed), pp 381-393. New York: Marcel Dekker.

Psarropoulou C, Dallaire F (1998) Activation of muscarinic receptors during blockade of GABA(A)-mediated inhibition induces synchronous epileptiform activity in immature rat hippocampus. Neuroscience 82:1067-1077.

Smidt CR, Unkefer CJ, Houck DR, Rucker RB (1991) Intestinal absorption and tissue distribution of [14C] pyrroloquinoline quinone in mice. Proc Soc Exp Biol Med 197:27-31.

Stuart GJ, Dodt H-U, Sakmann B (1993) Patch clamp recordings from the soma and dendrites of neurons in brain slices using infrared video microscopy. Pflügers Arch 423:511-518.

Sullivan JM, Traynelis SF, Chen HV, Escobar W, Heinemann SF, Lipton SA (1994) Identification of two cysteine residues that are required for redox modulation of the NMDA subtype of glutamate receptor. Neuron 13:929-936.

Tang LH, Aizenman E (1993) The modulation of $N$-methyl-Daspartate receptors by redox and alkylating reagents in rat cortical neurones in vitro. J Physiol (Lond) 465:303-323.

Tauck DL, Ashbeck GA (1990) Glycine synergistically potentiates the enhancement of LTP induced by a sulfhydryl reducing agent. Brain Res 419:129-132.

Tenny RT, Sharbrough FW, Anderson RE, Sundt Jr TM (1980) Correlation of intracellular redox states and $\mathrm{pH}$ with blood flow in primary and secondary seizure foci. Ann Neurol 8:564-573.

Tolliver JM, Pellmar TC (1987) Dithiothreitol elicits epileptiform activity in $\mathrm{CA}_{1}$ of the guinea pig hippocampal slice. Brain Res 404:133-141.

Wang C, Jensen FE (1996) Age dependence of NMDA receptor involvement in epileptiform activity in rat hippocampal slices. Epilepsy Res 23:105-113.

Williams JH, Kauer JA (1997) Properties of carbachol-induced oscillatory activity in rat hippocampus. J Neurophysiol 78:2631-2640. 